\title{
Detection of Global DNA Methylation in Cervical Intraepithelial Neoplasia and Cancerous Lesions by Pyrosequencing and Enzyme-Linked Immunosorbent Assays
}

\author{
Rungtip Thumbovorn ${ }^{1,2}$, Parvapan Bhattarakosol ${ }^{1,3}$, Arkom Chaiwongkot ${ }^{1,3 *}$
}

\begin{abstract}
Background: Cervical cancer is one of the most significant cancer found in women worldwide especially in developing countries. Previous reports showed that global DNA hypomethylation was correlated with various types of cancer including cervical cancer. Methods: Long interspersed nuclear element-1 (LINE1) pyrosequencing and Enzyme linked-immunosorbent assay (ELISA) assays were used for detection of global DNA methylation. The ELISA results were compared to bisulfite LINE1 pyrosequencing assay. Results: Different cervical cancer cell lines (CaSki, SiHa, HeLa, ME180, MS751, C33A) showed low global methylation percentage when compared to normal white blood cells by ELISA assay (1.47\%-5.09\% vs $8.20 \%$, respectively) and by LINE1 pyrosequencing (20\%- $45 \%$ vs $62 \%$, respectively). Global DNA methylation levels in cervical cancer samples were lower than precancerous lesions (Normal-CIN3) by LINE1 pyrosequencing (mean, $48.8 \%$ vs $56.9 \%$, respectively, $\mathrm{p}<0.05$ ) and ELISA assay (mean, $3.03 \%$ vs $3.85 \%$, respectively, $\mathrm{p}<0.05$ ). Conclusion: Global DNA hypomethylation was predominantly found in cervical cancer samples detected by ELISA and LINE1 pyrosequencing assays and could be used as triage tests in cervical cancer screening. ELISA assay is a suitable method for detection of global DNA methylation in large population; however, it should be further evaluated in a large clinical samples in order to be used as screening method.
\end{abstract}

Keywords: Global DNA methylation- enzyme linked immunosorbent assay- Long interspersed nuclear element-1

Asian Pac J Cancer Prev, 23 (1), 143-149

\section{Introduction}

Cervical cancer is one of the most common cancer found in women worldwide, approximately $>500,000$ cervical cancer cases were diagnosed annually and nearly $50 \%$ death (Torre et al., 2017). In low- and middle-income countries, cervical cancer is the second most frequently anogenital cancer found in women after breast cancer (Woodman et al., 2007; Torre et al., 2017).

Persistent infection with high risk or oncogenic human papillomavirus (HPV) types is accepted as a necessary cause of cervical cancer. Among high risk-HPV types, HPV 16 and HPV 18 cause $>70 \%$ of cervical cancers worldwide ( $\mathrm{Li}$ et al., 2011). HPV 16 is detected more than $50 \%$ in squamous cell carcinoma and HPV18 is mostly found in adenocarcinomas (Motoyama et al., 2004; Gnanamony et al., 2007). HPV 58 and 52 are mostly found in eastern Asian countries after HPV16 and 18 (Chan et al., 1999; Hwang, 1999; Chen et al., 2006; Inoue et al., 2006; Sukasem et al., 2011; Aromseree et al., 2014). However, the majority of HPV infected women has spontaneous regression, minor of them develop cancer (Melnikow et al.,
1998). Epigenetics changes are involved in carcinogenesis, one of these changes is global DNA hypomethylation of high repetitive DNA sequences such as long interspersed nuclear elements or LINE and Alu sequences (Ehrlich, 2009). Global DNA hypomethylation was found in most cancer types such as breast cancer (Wu et al., 2011) prostate cancer (Yegnasubramanian et al., 2008) bladder cancer (Choi et al., 2009) liver cancer (Takai et al., 2000) chronic myeloid leukemia (Roman-Gomez et al., 2008) and cervical cancer (Kim et al., 1994; Missaoui et al., 2010; Sen et al., 2017) that may have potential to be used as biomarker for screening of cancer including cancer of anogenital tracts.

Previous reports showed global DNA methylation in cervical cancer samples by detecting LINE1 gene methylation using combined bisulfite restriction analysis (COBRA) polymerase chain reaction and found association between LINE1 hypomethylation and cancer stage (Shuangshoti et al., 2007). Other group performed immunohistochemistry using antibody to 5-methylacytosine in formaline fixed paraffin embedded tissue (FFPE) and using high-performance capillary 
electrophoresis (HPCE), they found that global DNA hypomethylation was associated with cervical cancer (Missaoui et al., 2010).

Various assays have been used to detect global DNA methylation by detecting LINE1, Alu, Satellite-alpha and Satellite-2 regions using molecular assays such as real time methylation specific PCR, bisulfite sequencing PCR, combined bisulfite restriction analysis (COBRA) and pyrosequencing (Li et al., 2014; Kurdyukov and Bullock, 2016). However, these techniques need bisulfite treatment, require expensive equipments and are not suitable in low income countries for screening in general population. Therefore, we aimed to use enzyme linked immunosortbent assay (ELISA) that is more suitable to be used in low resource countries and high-throughout screening assay for detection of global methylation. DNA extracted from cervical cells samples with different lesions severity were employed. Results of ELISA assay were compared to bisulfite LINE1 pyrosequencing.

\section{Materials and Methods}

\section{Clinical samples and cell lines}

Left over cervical cells were collected from routine Pap smears at the Department of Gynecological Outpatient, King Chulalongkorn Memorial Hospital and the Department of Microbiology, Faculty of Medicine, Chulalongkorn University, Bangkok, Thailand. DNA was extracted from cervical cells by AllPrep DNA/RNA (Qiagen, Hilden, Germany). (In total, 280 samples were collected including normal $(\mathrm{n}=36)$, cervical intraepithelial neoplasia 1 (CIN1) $(n=115), C I N 2-3(n=39)$ and squamous cell carcinoma (SCC) $(n=90)$. Caski (500-600 copies of HPV16), SiHa (1-2 copies of HPV16), HeLa (HPV18 positive), ME180 (HPV39), MS751 (HPV45) and C33A (HPV negative) cell lines were used as cancerous cells control. White blood cells collected from a healthy donor was used as normal control. Buffy coat was removed from EDTA blood and used for DNA extraction using AllPrep DNA/RNA (Qiagen, Hilden, Germany). This study has been approved (COA No. 087/2016) by the Institutional Review Board of the Faculty of Medicine, Chulalongkorn University.

\section{Bisulfite conversion of DNA extracted from clinical samples} and LINE1 methylation analysis by pyrosequencing

DNA samples were converted using the EZ kit Gold Bisulfite Conversion Kit (Zymo Research) according to the manufacturer's instruction. The extracted DNA (100-1000 ng) (maximum 20 $\mu \mathrm{L}$ ) was used in bisulfite modification reaction. DNA extracted from human cervical cell lines (CaSki, SiHa, ME180, MS751 and C33A) were used as cancerous cells control and DNA extracted from normal white blood cellwas used as normal control for amplification and pyrosequencing.

The sequences of the LINE1 forward and reverse primers were FW: 5' - TTTTGAGTTAGGTGTGTGGGATATA-3' and RV: Biotin-5' - AAAATCAAAAAATTC CCTTTC-3' (150 bp). Sequencing primer and sequence to analyze were 5'- AGTTAGGT GTGGGATATAGT-3 and TGGTGYGTYGTTTTTTAAGTYGGTTTGAAAAGYA
TA, respectively (Delaney et al., 2015) . Bisulfite modified DNA samples were used for PCR amplification as follows: $13.6 \mu \mathrm{L}$ DNase/RNase free water, $1 \mathrm{X}$ PCR buffer, $2.5 \mathrm{mM} \mathrm{MgCl} 2,250 \mu \mathrm{M}$ dNTP, $12.5 \mathrm{pM}$ of each forward and reverse primers, 1 Unit DNA polymerase (HotStart HiFidelity Polymerase, Affymetrix, USA). The PCR conditions were as follows: initial denaturing at $95^{\circ} \mathrm{C}$ for 10 minutes, followed by 50 cycles of $95^{\circ} \mathrm{C}$ for 30 seconds, $50^{\circ} \mathrm{C}$ for 1 minute and $72^{\circ} \mathrm{C}$ for 30 seconds and final extension at $72^{\circ} \mathrm{C}$ for 5 minutes. The PCR products were detected by $1.5 \%$ agarose gel electrophoresis. Prior to pyrosequencing, $20 \mu \mathrm{L}$ of biotin labeled amplified products were mixed with beads, washed with $70 \%$ ethanol, denatured, washed and mixed with $0.4 \mu \mathrm{M}$ of sequencing primers and loaded into the PyroMark ${ }^{\mathrm{TM}}$ Q96 machine (Qiagen, Hilden, Germany).

Quantification of global DNA methylation by ELISAbased assay

ELISA was performed according to instruction manual (5-mC DNA ELISA Kit, Zymo Research)1. Briefly, 100 ng of DNA samples were denatured and placed on ice immediately for 10 minutes and subsequently applied to an ELISA well strip. After DNA-surface binding, primary antibody against 5-methyl cytosine and secondary antibody were applied in an ELISA strip well. After color development, the absorbance was read at $405 \mathrm{~nm}$ using a microplate reader (Perkin elmer Victor). The absolute quantity of global DNA methylation was determined by subtracting the sample OD from the negative control and calculate the percentage of methylation using equation achieved from a standard curve, $0 \%, 5 \%, 10 \%, 25 \%$, $50 \%, 75 \%$ and $100 \%$, which was obtained from positive control DNA with known 5-methyl cytosine concentration, running on the same plate in duplicate.

\section{Statistical analysis}

The Kruskal-Wallis test was used to analyze the differences of the mean methylation values among groups of specimens. Receiver operating characteristic (ROC) curve was used to analyze the sensitivity and specificity to diagnose lesion severity with cancerous lesion. P-value less than 0.05 was considered statistically significant.

\section{Results}

Methylation levels in cervical cancer cell lines

The optimal time and wavelength for absorbance measurement by ELISA assay were $40 \mathrm{~min}$ at $405 \mathrm{~nm}$. The results of global methylation percentage by ELISA found in various cervical cancer cell lines infected with different HPV types were as followed, 3.72\% (Caski), 1.74\% (SiHa), 2.42\% (HeLa), 4.79\% (ME189), 5.09\% (MS751) and 1.47\% (C33A) while DNA extracted from normal white blood cells showed $8.20 \%$. Bisulfite LINE1 pyrosequencing results were $45 \%$ of Caski, $35 \%$ of $\mathrm{SiHa}$, $20 \%$ of $\mathrm{C} 33 \mathrm{~A}$ and $62 \%$ of normal white blood cells. Both assays showed global DNA hypomethylation in cervical cancer cell lines when compared to normal white blood cells. 


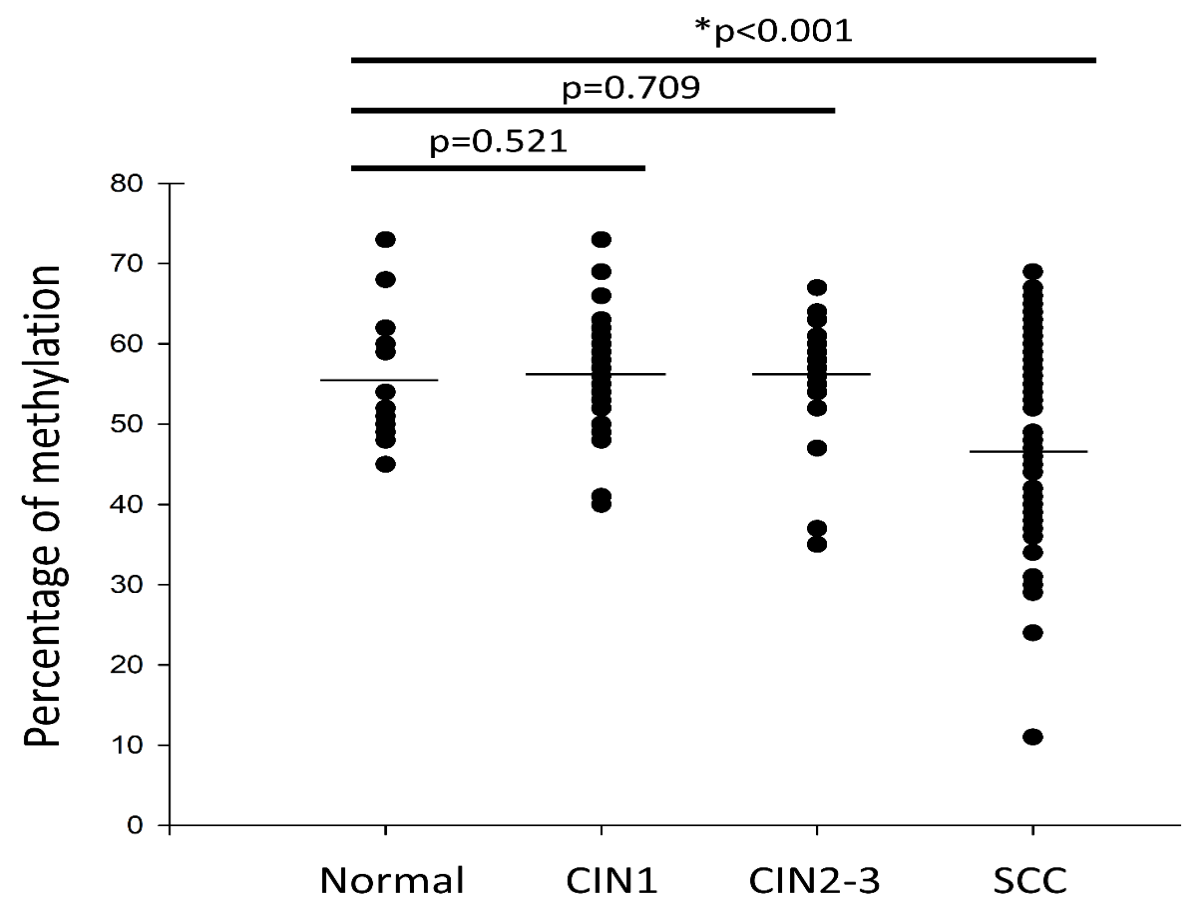

Figure 1.Methylation Levels in 167 Cervical Samples with Different Lesions Severity $(*=p<0.05$ (Kruskal wallis)).

\section{Methylation levels in clinical samples}

167 of 280 cervical samples were available for global DNA methylation by pyrosequencing and means of methylation were as followed; $55.6 \%$ of normal, $56.8 \%$ of CIN $1,57.3 \%$ of CIN $2-3$ and $48.8 \%$ of cervical cancer, there was a significant difference between cervical cancer samples and precancerous samples (normal-CIN3) $(\mathrm{p}<0.05)$ (Figure 1). 257 of 280 samples were used for global DNA methylation detection by ELISA assay, however, samples with optical density (OD) less than 0.1 were excluded. Ninety-seven cervical samples were included for further methylation analysis and methylation means were as followed, $4.50 \%$ of normal, 3.83 of CIN1, $2.83 \%$ of CIN2-3 and $3.03 \%$ of cervical cancer, there was a significant difference between cervical cancer samples and precancerous samples (normal-CIN3) $(\mathrm{p}<0.05)$ (Figure 2). Global DNA methylation level detected by ELISA assay was proportional to LINE1 pyrosequencing assay $(\mathrm{N}=58$, $r=0.442$, Figure 3).

Sensitivity and specificity of ELISA and LINE1 pyrosequencing assays for diagnosis of cervical cancerous lesions

We further analyzed whether the methylation level of a global DNA methylation by either bisulfite LINE1 pyrosequencing and ELISA assay could be used to predict cervical lesion severity. Receiver operating characteristic (ROC) curve was used to calculate the sensitivity and specificity at different methylation percentages as a cut off point to differentiate between precancerous and cancerous lesions. Figure 4 and 5 showed ROC curve and area under

Table 1. Sensitivity and Specificity of Global DNA Methylation to Differentiate between Precancerous (normal,CIN1,CIN2-3) and Cancerous Lesions by Bisulfite LINE1

\begin{tabular}{|c|c|c|c|c|c|c|c|c|}
\hline \multirow{3}{*}{ Cut off } & \multicolumn{8}{|c|}{ LINE1 pyrosequencing. } \\
\hline & \multicolumn{2}{|c|}{$30 \%$} & \multicolumn{2}{|c|}{$35 \%$} & \multicolumn{2}{|c|}{$40 \%$} & \multicolumn{2}{|c|}{$45 \%$} \\
\hline & Sensitivity & Specificity & Sensitivity & Specificity & Sensitivity & Specificity & Sensitivity & Specificity \\
\hline$\%$ & 4.76 & 100 & 9.52 & 100 & 23.81 & 97.59 & 36.9 & 95.18 \\
\hline \multirow[t]{2}{*}{ Cut off } & \multicolumn{2}{|c|}{$50 \%$} & \multicolumn{2}{|c|}{$55 \%$} & \multicolumn{2}{|c|}{$60 \%$} & \multicolumn{2}{|c|}{$65 \%$} \\
\hline & Sensitivity & Specificity & Sensitivity & Specificity & Sensitivity & Specificity & Sensitivity & Specificity \\
\hline \multirow[t]{2}{*}{$\%$} & 50 & 79.52 & 67.86 & 61.45 & 85.71 & 24.1 & 96.43 & 9.63 \\
\hline & \multicolumn{8}{|c|}{ ELISA } \\
\hline \multirow[t]{2}{*}{ Cut off } & \multicolumn{2}{|c|}{$1.50 \%$} & \multicolumn{2}{|c|}{$2.00 \%$} & \multicolumn{2}{|c|}{$2.50 \%$} & \multicolumn{2}{|c|}{$3.00 \%$} \\
\hline & Sensitivity & Specificity & Sensitivity & Specificity & Sensitivity & Specificity & Sensitivity & Specificity \\
\hline$\%$ & 17.86 & 91.3 & 28.57 & 78.26 & 42.86 & 60.87 & 57.14 & 56.62 \\
\hline \multirow[t]{2}{*}{ Cut off } & \multicolumn{2}{|c|}{$3.50 \%$} & \multicolumn{2}{|c|}{$4.00 \%$} & \multicolumn{2}{|c|}{$4.50 \%$} & \multicolumn{2}{|c|}{$5.00 \%$} \\
\hline & Sensitivity & Specificity & Sensitivity & Specificity & Sensitivity & Specificity & Sensitivity & Specificity \\
\hline$\%$ & 64.29 & 50.72 & 75 & 42.03 & 82.14 & 39.13 & 89.29 & 31.88 \\
\hline
\end{tabular}




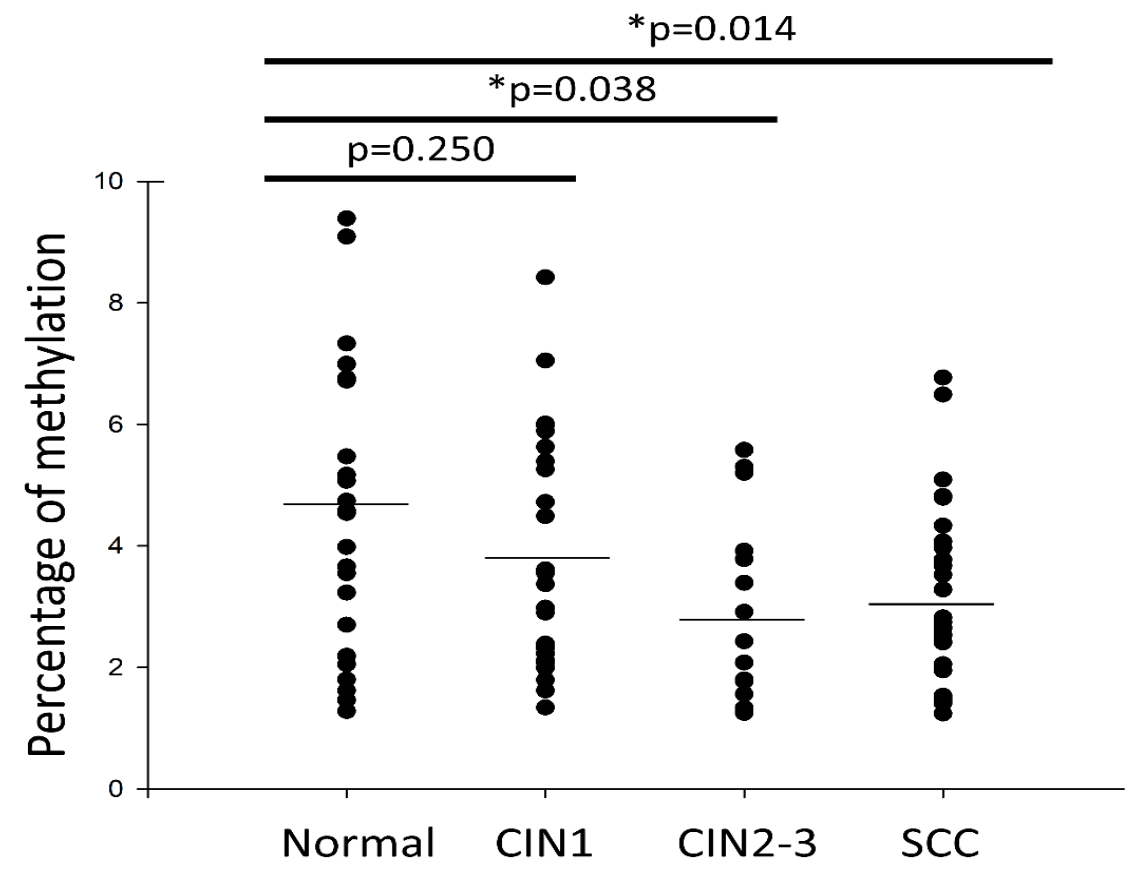

Figure 2. Methylation Levels in Ninety-Seven Cervical Samples with Different Lesions Severity Detected by ELISA assay $(*=p<0.05$ (Kruskal wallis)).

curve (AUC) of LINE1 pyrosequencing and ELISA (AUC $=0.72$ and 0.60 , respectively). At cut off points, $30 \%-45 \%$ of LINE1 pyrosequencing and $1.5 \%-2.0 \%$ of ELISA, revealed high specificity but low sensivity as shown in Table 1 , respectively.

\section{Discussion}

It has been shown that aberrant DNA methylation such as hypomethylation of human genome is associated with various types of cancer (Ehrlich, 2009). Various qualitative assays such as direct sequencing of PCR products or TA cloning of PCR products and quantitative assay such as pyrosequencing need bisulfite treatment to convert unmethylated cytosine to uracil but not 5-methycytosine (Kurdyukov and Bullock, 2016). ELISA based assay is the rough estimation of global DNA methylation, however, it is a quick and easy to perform assay and DNA can be directly detected without bisulfite treatment step. Furthermore, this assay is suitable for

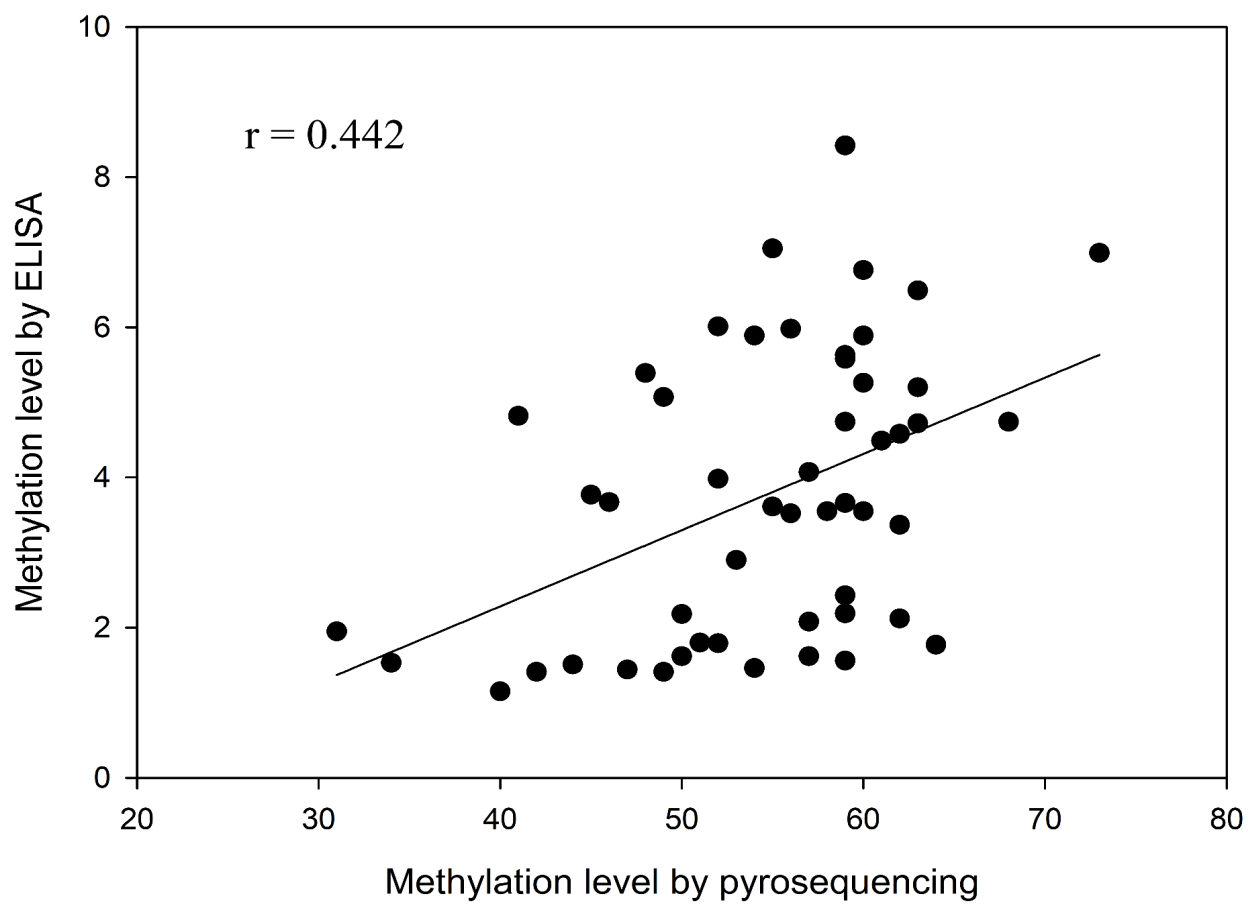

Figure 3. Correlation of Bisulfite LINE1 Pyrosequencing and ELISA Assays ( $\mathrm{N}=58)$. 


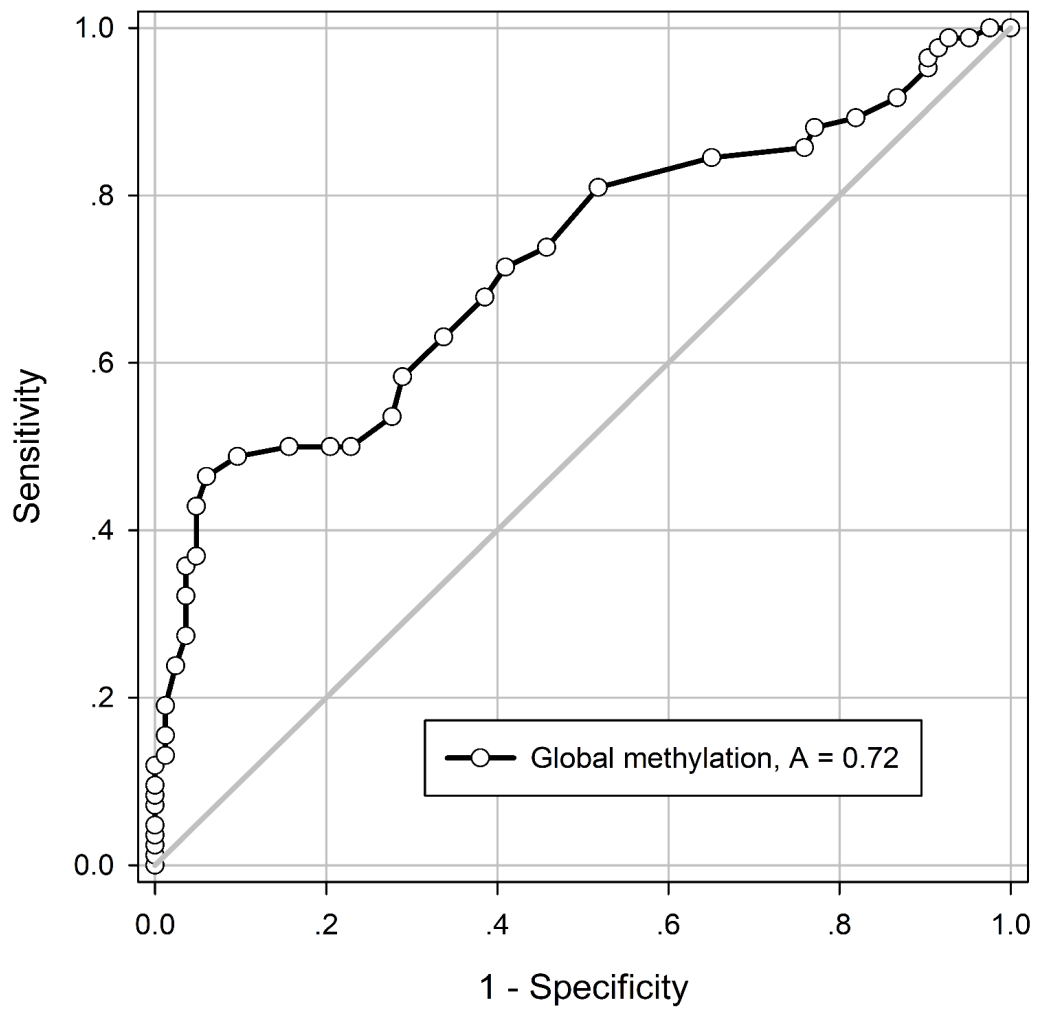

Figure 4. Receiver Operating Characteristic (ROC) Curve Shows the Sensitivity and Specificity of Methylation Levels to Differentiate between Precancerous (Normal,CIN1,CIN2-3) and Cancerous Lesions by Bisulfite LINE1 Pyrosequencing

high-throughput analysis of clinical samples escpeially in low resource countries because of its cost effectively (Kurdyukov and Bullock, 2016).
The present study employed bisulfite LINE1 pyrosequencing assay and ELISA based assay for analysis of global DNA methylation, the results indicated

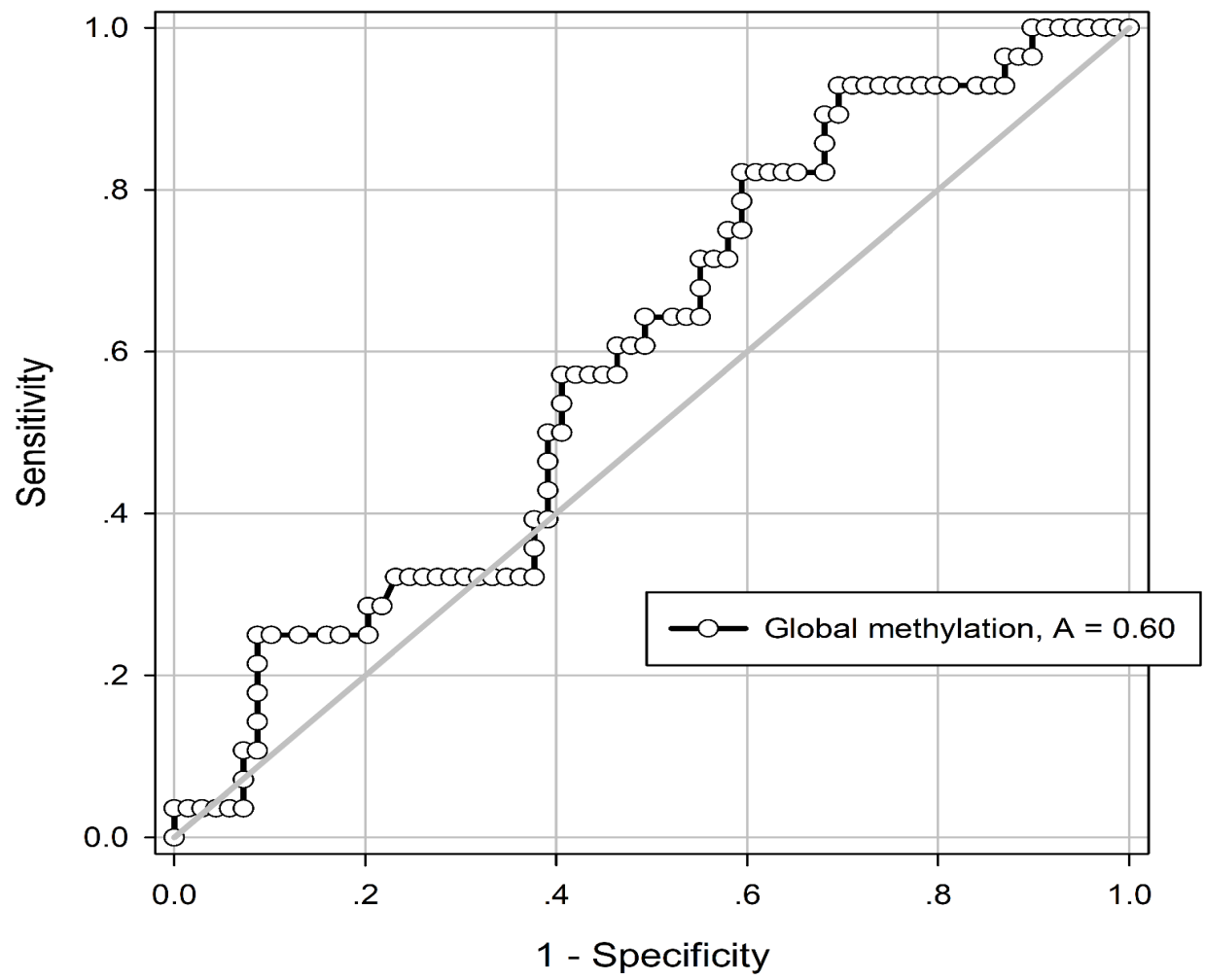

Figure 5. Receiver Operating Characteristic (ROC) Curve Shows the Sensitivity and Specificity of Methylation Levels to Differentiate between Precancerous (Normal,CIN1,CIN2-3) and Cancerous Lesions by ELISA Assay. 
progressive global DNA hypomethylation from normal cervical cells to cervical cancer samples by both assays. Our results were in accordant to previous studies in various cancer types (Flatley et al., 2009; Hou et al., 2010; Akers et al., 2014) . Akers et al. detected LINE1 methylation in ovarian cancer using bisulfite pyrosequencing and reported hypomethylation in epithelial ovarian cancer $(57.1 \%)$ when compared to normal ovarian surface epithelia (72.5\%) (Akers et al., 2014). Fotouhi et al reported LINE1 hypomethylation in small intestinal neuroendocrine tumors $(65 \%)$ compared to normal cells $(75 \%)$ by pyrosequencing and by ELISA assay, mean of methylation index in cancer samples was 1.1 when compared to methylation index 1.2 found in normal cells (Fotouhi et al., 2014). Foy et al found LINE1 hypomethylation in oral cancer compared non cancerous lesions (Foy et al., 2015). LINE1 hypomethylation was found in various types of cancer such as bladder, colon, pancreas, prostate and stomach cancers(Nüsgen et al., 2015). The study of global methylation in cervical cancer was reported using high-performance capillary electrophoresis (HPCE) assay, they reported hypomethylation in cancer cells that was 2.81 when compared to normal, low grade lesions and high grade lesions that were 3.26, 3.37 and 3.22, respectively, that suggested progressive hypomethylation (Missaoui et al., 2010). The other group reported progressive LINE1 hypomethylation level from normal epithelium to squamous cell carcinoma using combined bisulfite restriction analysis (COBRALINE1) PCR (Shuangshoti et al., 2007). The study using tritium-labeled methyl groups to incorporate into genomic DNA to detect global DNA methylation reported that most of cervical cancer samples had global DNA hypomethylation when compared to precancerous lesions(Flatley et al., 2009).

This study has some limitaions, HPV DNA detection and typing was not performed in the present study, therefore, correlation between HPV status and global DNA hypomethylaltion could not be analysed. One study reported that global DNA hypomethylation was mostly found in HPV16 infected cervical cancer samples when compared to HPV negative normal control. Moreover, it was reported that HPV16 episomal form was correlated with global DNA hypomethylation than HPV16 integration form (Sen et al., 2017). However, one study in head and neck cancer showed contrary results, they reported LINE-1 hypomethylation was mostly found in HPV-negative than in HPV-positive cancer samples(Richards et al., 2009; van Kempen et al., 2014). In addition, LINE-1 hypomethylation was correlated with genomic instability(Richards et al., 2009). The DNA needed in ELISA reaction was $100 \mathrm{ng}$, nevertheless, input DNA used was less than $100 \mathrm{ng}$, therefore more than half of samples were excluded for further data analysis due to very low optical density $(<0.1)$ that similar to negative control. In addition, the quantification of global DNA methylation level by ELISA assay $(<10 \%)$ was particularly lower than results of LINE1 pyrosequencing assay. However, previous study used the same ELISA kit reported the similar percentage of global DNA methylation $(<10 \%)$ compared to LINE1 methylation (Fa et al., 2016).
Signal amplification using horseradish peroxidase (HRP) enzyme system may be the reason of low global DNA methylation level compared to target amplification assay of LINE1 bisulfite pyrosequencing.

In conclusion, global DNA hypomethylation quantified by ELISA assay was at least correlated with quantitative bisulfite LINE1 pyrosequencing in term of progressive global DNA hypomethylation. ELISA based assay should be further developed and evaluated in large clinical samples size in order to obtain suitable cut off point and to be used as triage test in cervical cancer screening to differentiate women who are at high risk to develop cervical cancer quickly from low risk women.

\section{Author Contribution Statement}

AC designed the experiments and was a major contributor to writing the manuscript. RT performed the experiments. PB supervised the project and reviewed the manuscript.

\section{Acknowledgements}

This Research is funded by Chulalongkorn university; Government Budget (grant number GRB_ BSS_78_59_30_19).

\section{Data Availability Statement}

Data supporting the findings of the present study are available within the article. Raw data that support the findings of the present study are available from the corresponding author, upon reasonable request.

\section{Ethical approval}

This study has been approved (COA No. 087/2016) by the Institutional Review Board of the Faculty of Medicine, Chulalongkorn University. This article was not a part of student thesis.

\section{Conflict of Interest}

The authors declare that there is no conflict of interest.

\section{References}

Akers SN, Moysich K, Zhang W, et al (2014). LINE1 and Alu repetitive element DNA methylation in tumors and white blood cells from epithelial ovarian cancer patients. Gynecol Oncol, 132, 462-7.

Aromseree S, Chaiwongkot A, Ekalaksananan T, et al (2014). The three most cosmmon human papillomavirus oncogenic types and their integration state in Thai women with cervical precancerous lesions and carcinomas. J Med Virol, 86, 1911-9.

Chan PK, Li WH, Chan MY, et al (1999). High prevalence of human papillomavirus type 58 in Chinese women with cervical cancer and precancerous lesions. J Med Virol, 59, 232-8.

Chen CA, Liu CY, Chou HH, et al (2006). The distribution and differential risks of human papillomavirus genotypes in cervical preinvasive lesions: A Taiwan Cooperative Oncologic Group Study. Int J Gynecol Cancer, 16, 1801-8. Choi SH, Worswick S, Byun HM, et al (2009). Changes in DNA 
methylation of tandem DNA repeats are different from interspersed repeats in cancer. Int J Cancer, 125, 723-9.

Delaney C, Garg SK, Yung R (2015). Analysis of DNA Methylation by Pyrosequencing. Methods Mol Biol, 1343, 249-64.

Ehrlich M (2009). DNA hypomethylation in cancer cells. Epigenomics, 1, 239-59.

Fa S, Larsen TV, Bilde K, et al (2016). Assessment of global DNA methylation in the first trimester fetal tissues exposed to maternal cigarette smoking. Clin Epigenetics, 8, 128.

Flatley JE, McNeir K, Balasubramani L, et al (2009). Folate status and aberrant DNA methylation are associated with HPV infection and cervical pathogenesis. Cancer Epidemiol Biomarkers Prev, 18, 2782-9.

Fotouhi O, Adel Fahmideh M, Kjellman M, et al (2014). Global hypomethylation and promoter methylation in small intestinal neuroendocrine tumors: An in vivo and in vitro study. Epigenetics, 9, 987-97.

Foy JP, Pickering CR, Papadimitrakopoulou VA, et al (2015). New DNA methylation markers and global DNA hypomethylation are associated with oral cancer development. Cancer Prev Res (Phila), 8, 1027-35.

Gnanamony M, Peedicayil A, Abraham P (2007). An overview of human papillomaviruses and current vaccine strategies. Indian J Med Microbiol, 25, 10-7.

Hou L, Wang H, Sartori S, et al (2010). Blood leukocyte DNA hypomethylation and gastric cancer risk in a high-risk Polish population. Int J Cancer, 127, 1866-74.

Hwang T (1999). Detection and typing of human papillomavirus DNA by PCR using consensus primers in various cervical lesions of Korean women. J Korean Med Sci, 14, 593-9.

Inoue M, Sakaguchi J, Sasagawa T, et al (2006). The evaluation of human papillomavirus DNA testing in primary screening for cervical lesions in a large Japanese population. Int $J$ Gynecol Cancer, 16, 1007-13.

Kim YI, Giuliano A, Hatch KD, et al (1994). Global DNA hypomethylation increases progressively in cervical dysplasia and carcinoma. Cancer, 74, 893-9.

Kurdyukov S, Bullock M (2016). DNA Methylation Analysis: Choosing the Right Method. Biology (Basel), 5.

Li J, Huang Q, Zeng F, et al (2014). The prognostic value of global DNA hypomethylation in cancer: a meta-analysis. PLoS One, 9, e106290.

Li N, Franceschi S, Howell-Jones R, et al (2011). Human papillomavirus type distribution in 30,848 invasive cervical cancers worldwide: Variation by geographical region, histological type and year of publication. Int $J$ Cancer, 128, 927-35.

Melnikow J, Nuovo J, Willan AR, et al (1998). Natural history of cervical squamous intraepithelial lesions: a meta-analysis. Obstet Gynecol, 92, 727-35.

Missaoui N, Hmissa S, Dante R, et al (2010). Global DNA methylation in precancerous and cancerous lesions of the uterine cervix. Asian Pac J Cancer Prev, 11, 1741-4.

Motoyama S, Ladines-Llave CA, Luis Villanueva S, et al (2004). The role of human papilloma virus in the molecular biology of cervical carcinogenesis. Kobe J Med Sci, 50, 9-19.

Nüsgen N, Goering W, Dauksa A, et al (2015). Inter-locus as well as intra-locus heterogeneity in LINE-1 promoter methylation in common human cancers suggests selective demethylation pressure at specific CpGs. Clin Epigenetics, 7, 17.

Richards KL, Zhang B, Baggerly KA, et al (2009). Genomewide hypomethylation in head and neck cancer is more pronounced in HPV-negative tumors and is associated with genomic instability. PLoS One, 4, e4941.

Roman-Gomez J, Jimenez-Velasco A, Agirre X, et al (2008). Repetitive DNA hypomethylation in the advanced phase of chronic myeloid leukemia. Leuk Res, 32, 487-90.

Sen S, Mandal P, Bhattacharya A, et al (2017). Impact of viral and host DNA methylations on HPV16-related cervical cancer pathogenesis. Tumour Biol, 39, 1010428317699799.

Shuangshoti S, Hourpai N, Pumsuk U, et al (2007). Line-1 hypomethylation in multistage carcinogenesis of the uterine cervix. Asian Pac J Cancer Prev, 8, 307-9.

Sukasem C, Pairoj W, Saekang N, et al (2011). Molecular epidemiology of human papillomavirus genotype in women with high-grade squamous intraepithelial lesion and cervical cancer: Will a quadrivalent vaccine be necessary in Thailand?. J Med Virol, 83, 119-26.

Takai D, Yagi Y, Habib N, et al (2000). Hypomethylation of LINE1 retrotransposon in human hepatocellular carcinomas, but not in surrounding liver cirrhosis. Jpn J Clin Oncol, 30, 306-9.

Torre LA, Islami F, Siegel RL, et al (2017). Global cancer in women: burden and trends. Cancer Epidemiol Biomarkers Prev, 26, 444-57.

Van Kempen PM, Noorlag R, Braunius WW, et al (2014). Differences in methylation profiles between HPV-positive and HPV-negative oropharynx squamous cell carcinoma: a systematic review. Epigenetics, 9, 194-203.

Woodman CB, Collins SI, Young LS (2007). The natural history of cervical HPV infection: unresolved issues. Nat Rev Cancer, 7, 11-22.

Wu HC, John EM, Ferris JS, et al (2011). Global DNA methylation levels in girls with and without a family history of breast cancer. Epigenetics, 6, 29-33.

Yegnasubramanian S, Haffner MC, Zhang Y, et al (2008). DNA hypomethylation arises later in prostate cancer progression than $\mathrm{CpG}$ island hypermethylation and contributes to metastatic tumor heterogeneity. Cancer Res, 68, 8954-67.

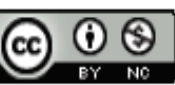

This work is licensed under a Creative Commons AttributionNon Commercial 4.0 International License. 